TRANSACTIONS OF THE

AMERICAN MATHEMATICAL SOCIETY

Volume 356, Number 3, Pages 1007-1024

S 0002-9947(03)03181-7

Article electronically published on August 25, 2003

\title{
THE 2-TWIST-SPUN TREFOIL HAS THE TRIPLE POINT NUMBER FOUR
}

\author{
SHIN SATOH AND AKIKO SHIMA
}

\begin{abstract}
The triple point number of an embedded surface in 4-space is the minimal number of the triple points on all the projection images into 3-space. We show that the 2-twist-spun trefoil has the triple point number four.
\end{abstract}

\section{INTRODUCTION}

Throughout this paper, we work in the piecewise linear category. By a surface$k n o t$, we mean a connected or disconnected closed surface embedded in the 4dimensional Euclidean space $\mathbb{R}^{4}$ locally flatly. Two surface-knots are equivalent if they are related by an ambient isotopy of $\mathbb{R}^{4}$. A projection $\pi: \mathbb{R}^{4} \rightarrow \mathbb{R}^{3}$ is generic for a surface-knot $F$ if the image $\pi(F)$ in $\mathbb{R}^{3}$ is locally homeomorphic to (i) a single sheet, (ii) two transversely intersecting sheets, (iii) three transversely intersecting sheets, or (iv) a cross-cap. The points corresponding to (ii), (iii) and (iv) are called a double point, a triple point, and a branch point of the generic projection respectively. See Figure 1. The set of those points is called the singularity set of the generic projection. The (minimal) triple point number of a surface-knot $F$, denoted by $\mathrm{t}(F)$, is the minimal number of triple points of generic projections for all surface-knots which are equivalent to $F$. We remark that the minimal branch point number of a surface-knot can be defined similarly and is completely determined in terms of the normal Euler number of the surface-knot [1, 8].

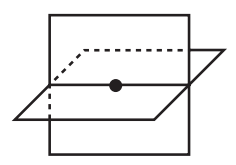

a double point

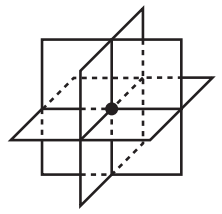

a triple point

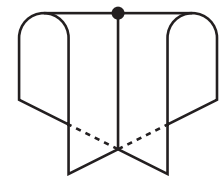

a branch point

FiguRE 1.

The notion of triple point numbers of surface-knots is an analogue to that of crossing numbers of classical knots ('classical' means embedded circles in $\mathbb{R}^{3}$ ). For classical knots, we have many examples whose crossing numbers are determined.

Received by the editors October 15, 2001 and, in revised form, July 24, 2002.

2000 Mathematics Subject Classification. Primary 57Q45; Secondary 57Q35.

Key words and phrases. 2-knot, surface-knot, triple point, cocycle invariant, motion picture. 
On the other hand, for surface-knots, there are not so many examples whose triple point numbers are determined. As examples of surface-knots whose triple point numbers are determined, the first author constructed a 2-component surface-link (a disconnected surface-knot) $F_{n}=F_{n}^{1} \cup F_{n}^{2}$ for any positive integer $n$ such that each connected component $F_{n}^{i}(i=1,2)$ is a non-orientable closed surface and $\mathrm{t}\left(F_{n}\right)=2 n$ [30].

A surface-knot is called a 2 -knot if it is an embedding of a 2-sphere. Two important families of 2-knots are ribbon 2-knots and twist-spins of classical knots. In 34], Yajima proved that a 2 -knot $F$ is a ribbon 2-knot if and only if $\mathrm{t}(F)=0$ (an alternative proof is found in [17]). On the other hand, the $m$-twist-spin of a classical knot $K$, denoted by $\tau^{m} K(m \geq 0)$, is a possibly non-ribbon 2-knot. More precisely, $\tau^{m} K$ is a ribbon 2-knot if and only if $m=0,1$ or $K$ is trivial [11; in particular, $\tau^{1} K$ is a trivial 2-knot for any $K$ [35. (see also [14, 22]). Hence, we have $\mathrm{t}\left(\tau^{m} K\right) \geq 2$ for any $m \geq 2$ and non-trivial $K$; it is known that every surface-knot $F$ satisfies $\mathrm{t}(F) \neq 1[28$.

However, till now, there have been no examples of non-ribbon twist-spins or 2knots in general whose triple point numbers are determined concretely. The aim of this paper is to prove the following.

Theorem 1.1. The 2-twist-spun trefoil has the triple point number four.

In [32, the second author proves that if a generic projection of a 2-knot $F$ has two triple points and no branch points, then $F$ is a ribbon 2-knot. However, this does not imply that $\mathrm{t}(F) \geq 3$ for any non-ribbon 2-knot $F$ immediately; for, in our definition of triple point numbers, generic projections are allowed to have finitely many branch points in general.

A quandle [16, 23] is a generalization of a group (under conjugation, so $a * b=$ $\left.b^{-1} a b\right)$ which is defined to be a set $Q$ with a binary operation $*: Q \times Q \rightarrow Q$ such that (i) $a * a=a$ for any $a \in Q$, (ii) for any $a, b \in Q$, there exists $x \in Q$ uniquely satisfying $x * a=b$, and (iii) $(a * b) * c=(a * c) *(b * c)$ for any $a, b, c \in Q$. The quandle cohomology group $H^{*}(Q ; G)$ is defined for a quandle $Q$ and an Abelian group $G$ in 3 . Then each third cohomology class $[\theta] \in H^{3}(Q ; G)$ gives an invariant of oriented surface-knots valued in the group ring $\mathbb{Z}[G]$,

$$
\Phi_{\theta}:\{\text { oriented surface-knots }\} \rightarrow \mathbb{Z}[G] .
$$

For an oriented surface-knot $F, \Phi_{\theta}(F)$ is called the cocycle invariant of $F$ with respect to the 3 -cocycle $\theta[3$. Further studies are found in $2,4,4,5,6,7,15,21,27,31$ and [33], for example. The set $\{0,1, \ldots, n-1\}$ with the operation $a * b=2 b-a$ $(\bmod n)$ forms a quandle, which is called the dihedral quandle [12] of order $n$, and is denoted by $R_{n}$. Then we have the following.

Theorem 1.2. Let $\theta$ be a 3-cocycle of the dihedral quandle $R_{3}$ with a coefficient group $G$. If the cocycle invariant $\Phi_{\theta}(F) \in \mathbb{Z}[G]$ of an oriented surface-knot $F$ satisfies $\Phi_{\theta}(F) \notin \mathbb{Z} \subset \mathbb{Z}[G]$, then we have $\mathrm{t}(F) \geq 4$.

In the condition of Theorem $1.2, \mathbb{Z} \subset \mathbb{Z}[G]$ means the subring $\left\{n \cdot 1_{G} \mid n \in \mathbb{Z}\right\}$ of $\mathbb{Z}[G]$, where $1_{G}$ is the identity of $G$. By taking $G$ as $\mathbb{Z}_{n}=\left\langle t \mid t^{n}=1\right\rangle$, we identify $\mathbb{Z}[G]$ with $\Lambda_{n}=\mathbb{Z}\left[t, t^{-1}\right] /\left(t^{n}-1\right)$. It is known that the 2-twist-spun trefoil $\tau^{2}$ (trefoil) has the cocycle invariant $\Phi_{\theta}\left(\tau^{2}\right.$ (trefoil)) $=3+6 t^{2} \in \Lambda_{3}$ for a 3 -cocycle $\theta$ with $[\theta] \in H^{3}\left(R_{3} ; \mathbb{Z}_{3}\right)$ [3, 7, 31. Since $\Phi_{\theta}\left(\tau^{2}\right.$ (trefoil) $) \notin \mathbb{Z} \subset \Lambda_{3}$, we have $\mathrm{t}\left(\tau^{2}(\right.$ trefoil $\left.)\right) \geq 4$ by Theorem 1.2. 
In order to estimate the triple point number of a surface-knot (in particular, a twist-spin) from above, we may give its generic projection explicitly. First of all, we recall the definition of twist-spins [35]. We think of $\mathbb{R}^{4}$ as an open book decomposition $\mathbb{R}_{+}^{3} \times S^{1}$ with $\{x\} \times S^{1}$ identified with $x$ for each $x \in \mathbb{R}^{2}=\partial \mathbb{R}_{+}^{3}$; that is, spinning $\mathbb{R}_{+}^{3}$ about $\mathbb{R}^{2}$ generates $\mathbb{R}^{4}$. We take a tangle $T$ in $\mathbb{R}_{+}^{3}$ whose knotting is a classical knot $K$. Then the $m$-twist-spin $\tau^{m} K$ of $K$ is obtained by spinning $T \subset \mathbb{R}_{+}^{3}$ about $\mathbb{R}^{2}$ as we simultaneously twist $T m$ times. To get a generic projection of $\tau^{m} K$, we think of $\mathbb{R}^{3}$ as an open book decomposition $\mathbb{R}_{+}^{2} \times S^{1}$ with a similar identification for each $x \in \mathbb{R}^{1}=\partial \mathbb{R}_{+}^{2}$. Let $\pi: \mathbb{R}^{4} \rightarrow \mathbb{R}^{3}$ be the projection given by a projection $\mathbb{R}_{+}^{3} \rightarrow \mathbb{R}_{+}^{2}$ crossed with the identity on $S^{1}$. Then the image $\pi\left(\tau^{m} K\right)$ is described by a series of tangle diagrams in $\mathbb{R}_{+}^{2} \times\{t\}\left(t \in S^{1}\right)$ corresponding to embeddings in $\mathbb{R}_{+}^{3} \times\{t\}$. Such a series of tangle diagrams is called a motion picture in the sense of [4, 27, 31. It is known that each Reidemeister move III in a motion picture produces a triple point of the corresponding generic projection. Hence, we may count the number of Reidemeister move III's instead of the number of triple points. For example, if $K$ has a knot diagram with $c$ crossings, then we can describe a motion picture corresponding to one twist of $T$ with $2(c-1)$ Reidemeister move III's. This implies that $\mathrm{t}\left(\tau^{m} K\right) \leq 2(c-1) m$ [31]. In this paper, we improve this result under some conditions as follows.

Theorem 1.3. If a classical knot $K$ has a knot diagram with $c$ crossings in which there is a pair of crossings as shown in Figure 2 , then we have $\mathrm{t}\left(\tau^{m} K\right) \leq 2(c-2) m$.

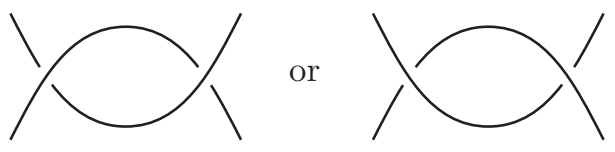

FIGURE 2 .

Theorem 1.3 is proved by giving a motion picture corresponding to one twist of $T$ with 2(c-2) Reidemeister move III's. Since the trefoil has a knot diagram with $c=3$ satisfying the condition in Theorem 1.3 , we have $\mathrm{t}\left(\tau^{2}\right.$ (trefoil $\left.)\right) \leq 2 \cdot(3-2) \cdot 2=4$. Thus, Theorem 1.1 follows from Theorems 1.2 and 1.3 immediately.

For a classical knot, we often use a knot diagram which is a regular projection with crossing information at the double points. Similarly, a surface diagram of a surface-knot is a generic projection with crossing information at the singularity set. For a surface-knot, we will use surface diagrams instead of generic projections. In Section 2, we review three fundamental properties on the singularity set of a surface diagram (Lemmas 2.1-2.3). In Section 3, we introduce the notion of tricolorings for a surface diagram as well as for a classical knot diagram [13. When a tricolored surface diagram is oriented, we define colors of double points and triple points derived from the colors of the sheets around them. Such colors enable us to obtain further properties on the singularity set of a tricolored surface diagram (Lemmas 3.2 and 3.4). In Section 4, we prove three key propositions for the proof of Theorem 1.2 (Propositions 4.1-4.3). Section 5 is devoted to proving Theorem 1.2. In Section 6 , we review the motion picture of a twist-spin given in [31] and prove Theorem 1.3. In [24], Mochizuki proves that $H^{3}\left(R_{p} ; \mathbb{Z}_{p}\right) \cong \mathbb{Z}_{p}$ for any prime $p>2$ and gives an explicit presentation of its generator. Section 7 is devoted to demonstrating 
that the 2-twist-spun trefoil has the cocycle invariant $3+6 t^{2} \in \Lambda_{3}$ with respect to Mochizuki's 3-cocycle for $p=3$.

The second author is partially supported by Grant-in-Aid for Scientific Research (No.12640090), Ministry of Education, Science and Culture, Japan.

\section{Preliminaries}

A surface-knot is a connected or disconnected closed surface embedded in $\mathbb{R}^{4}$ locally flatly. In particular, a surface-knot is oriented if each connected component is an oriented closed surface. A projection $\pi: \mathbb{R}^{4} \rightarrow \mathbb{R}^{3}$ is generic for a surface-knot $F$ if the singularity set of $\pi(F)$ consists of double points, isolated triple points, and isolated branch points. A surface diagram of $F$ is the image $\pi(F)$ with additional crossing information at the singularity set. See [10] or the beginning of Section 3 for more details. The singularity set of a surface diagram is regarded as a disjoint union of

(i) a graph with 1- and 6-valent vertices which correspond to branch points and triple points respectively, and

(ii) circles without self-intersections.

The above graph and circles may be linked in $\mathbb{R}^{3}$. An edge of the surface diagram is an edge of the graph in (i) or a circle in (ii). Figure 3 shows an example of a singularity set. Note that the number of branch points in each connected component of a singularity set is always even.
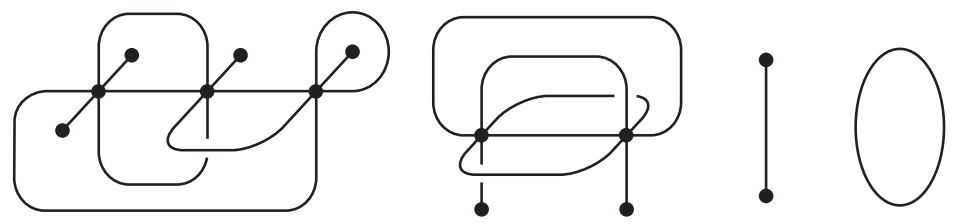

FIGURE 3.

In the following Lemmas 2.1-2.3, we do not assume that surface-knots and surface diagrams are oriented.

Lemma 2.1. If a surface diagram has an odd number of triple points, then there are at least two edges such that the endpoints of each edge are a branch point and a triple point.

Proof. There is a connected component of the singularity set, say $G_{0}$, in which the number of triple points is odd. We denote by $B_{0}$ and $T_{0}$ the numbers of branch points and triple points in $G_{0}$, respectively. Since $G_{0}$ has at least one triple point, it is sufficient to prove that $B_{0} \geq 2$. Assume that $B_{0}=0$. We recall that any singularity set admits an orientation, called a $B W$ orientation 29], such that the six edges at each triple point are oriented as shown in Figure 4(i) or (ii). For a fixed BW orientation of $G_{0}$, we denote by $T_{1}$ and $T_{2}$ the numbers of triple points in $G_{0}$ as in (i) and (ii) respectively. By counting the initials and terminals of the oriented edges, we have $2 T_{1}+4 T_{2}=4 T_{1}+2 T_{2}$, and hence $T_{0}=T_{1}+T_{2} \equiv 0(\bmod$ 2 ). This contradicts the assumption. Hence, we have $B_{0} \geq 1$. Since $B_{0}$ is even, we have $B_{0} \geq 2$. 


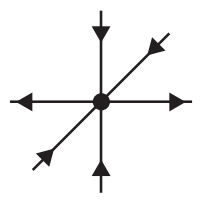

(i)

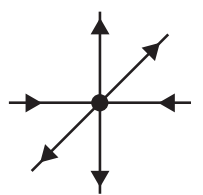

(ii)

FiguRE 4.

Let $\tau$ be a triple point of a surface diagram. There are three sheets around $\tau$ labeled top, middle and bottom with respect to the projection direction of $\pi: \mathbb{R}^{4} \rightarrow$ $\mathbb{R}^{3}$. Let $e$ be an edge which connects to $\tau$. The edge $e$ is called a $b / m-, b / t$ - or $m / t$-edge at $\tau$ if $e$ is the intersection of bottom and middle, bottom and top, or middle and top sheets around $\tau$, respectively.

As well as Reidemeister moves on knot diagrams, we have fundamental deformations, called Roseman moves [25], on surface diagrams such that two surface diagrams present equivalent surface-knots if and only if they are related by a finite sequence of Roseman moves.

Lemma 2.2 (cf. 28, 29]). Let e be an edge of a surface diagram whose endpoints are a branch point and a triple point $\tau$. If $e$ is a $b / m$ - or $m / t$-edge at $\tau$, then we can remove $\tau$ by some Roseman moves in a neighborhood of $e$.

Proof. Let $H$ be the sheet at $\tau$ which is transverse to $e$. By assumption, $H$ is a top or bottom sheet at $\tau$. Hence, we can perform a deformation on $H$ as shown in Figure 5, where $H$ is indicated by the shaded sheet. We see that this deformation is a combination of some Roseman moves.

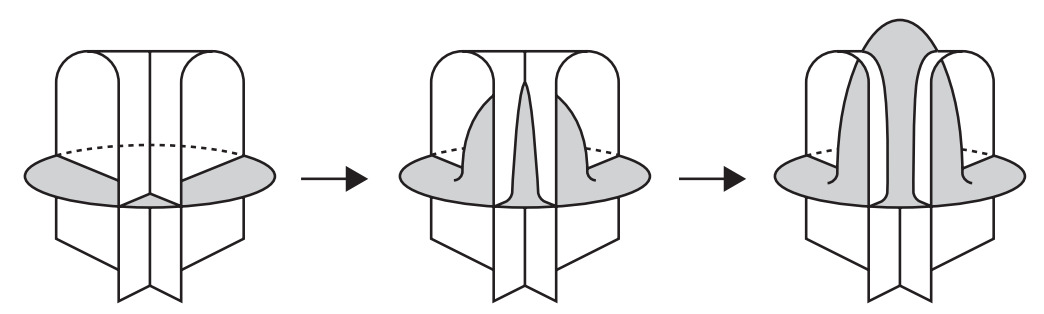

FigURE 5 .

Let $e_{1}, \ldots, e_{n}$ and $\tau_{1}, \ldots, \tau_{n}$ be edges and triple points of a surface diagram respectively such that the endpoints of $e_{i}$ are $\tau_{i}$ and $\tau_{i+1}$ and that $e_{i}$ and $e_{i+1}$ are in opposition to each other at $\tau_{i+1}(i=1, \ldots, n)$, where we take $e_{n+1}=e_{1}$ and $\tau_{n+1}=\tau_{1}$. Then the union $L=e_{1} \cup \cdots \cup e_{n}$ is called a cycle of the surface diagram. Recall that a circle without triple points is also called an edge of a surface diagram. We may include such circles in the set of cycles. Then we have the following.

Lemma 2.3 (29]). The number of triple points on each cycle is even.

Proof. Let $L=e_{1} \cup \cdots \cup e_{n}$ be a cycle of a surface diagram as above. We give a BW orientation to the singularity set. By the definition of a BW orientation as shown in Figure 4, we see that $e_{i}$ and $e_{i+1}$ have opposite orientations on both sides of $\tau_{i+1}(i=1, \ldots, n)$. Hence, $n$ is even. See Figure 6 . 


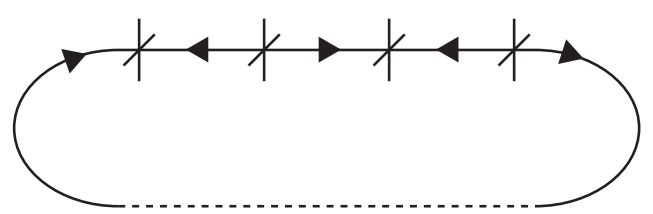

FiguRE 6.

\section{TRICOLORINGS FOR SURFACE DIAGRAMS}

Recall that a surface diagram of a surface-knot $F$ is an image $\pi(F)$ under a generic projection $\pi: \mathbb{R}^{4} \rightarrow \mathbb{R}^{3}$ with crossing information at the singularity set. There are two intersecting sheets along each edge, one of which is higher than the other with respect to $\pi$. They are called an over-sheet and an under-sheet along the edge, respectively. In order to indicate crossing information, we break the undersheet into two pieces missing the over-sheet. This modification is extended to a triple point such that the top sheet is not broken and the middle (or bottom) sheet is broken into two (or four) pieces. Then the surface diagram is presented by a disjoint union of compact surfaces which are called broken sheets. Refer to [10].

In the consecutive sections, all the surface-knots are assumed to be oriented, and their surface diagrams are also oriented coherently. For a surface diagram $D$, let $\mathcal{B}(D)$ denote the set of the broken sheets of $D$. Along each edge of the singularity set, there exist broken sheets $H_{1}, H_{2}, H_{1}^{\prime} \in \mathcal{B}(D)$ uniquely (some of which possibly coincide) such that

- $H_{2}$ is the over-sheet, and

- $H_{1}$ (or $H_{1}^{\prime}$ ) is the under-sheet such that it is in back (or front) of $H_{2}$ with respect to the orientation of $\mathrm{H}_{2}$.

See the left of Figure 7. In the figure, we indicate the orientations of sheets by arrows.
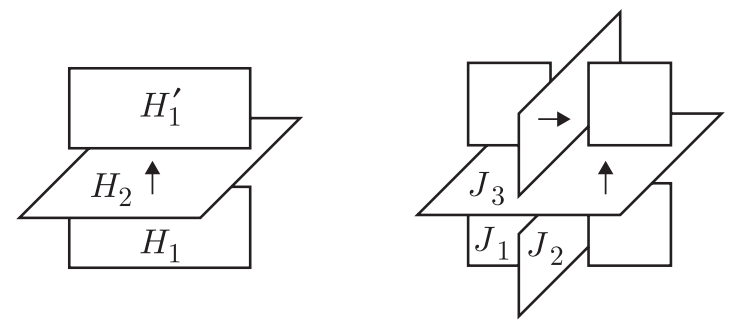

FiguRE 7.

We denote by $R_{3}$ the dihedral quandle of order 3 , which is the set $\{0,1,2\}$ with the binary operation $a * b=2 b-a(\bmod 3)$. A tricoloring for a surface diagram $D$ is a map $C: \mathcal{B}(D) \rightarrow R_{3}$ such that

$$
C\left(H_{1}\right) * C\left(H_{2}\right)=C\left(H_{1}^{\prime}\right)
$$

along every edge of $D$.

Definition 3.1. In the notation above, the color of each edge is the pair

$$
\left(C\left(H_{1}\right), C\left(H_{2}\right)\right) \in R_{3} \times R_{3} .
$$


An edge is called degenerate if $C\left(H_{1}\right)=C\left(H_{2}\right)$, and otherwise nondegenerate.

We remark that the definition of a tricoloring is equivalent to

$$
C\left(H_{1}\right)=C\left(H_{2}\right)=C\left(H_{1}^{\prime}\right) \text { or }\left\{C\left(H_{1}\right), C\left(H_{2}\right), C\left(H_{1}^{\prime}\right)\right\}=\{0,1,2\} .
$$

Hence, tricolorings for a surface diagram can be defined even if the diagram is not oriented. The orientation of a surface diagram (in particular, that of an over-sheet) is used for the definition of the color of an edge. If we reverse the orientation of a tricolored surface diagram, then the color of each edge changes into $\left(C\left(H_{1}^{\prime}\right), C\left(H_{2}\right)\right)$ but the degeneracy does not change.

Lemma 3.2. Let e be an edge of a tricolored surface diagram. If one of the endpoints of $e$ is a branch point, then e is degenerate.

Proof. Since $e$ connects to a branch point, all the three sheets $H_{1}, H_{1}^{\prime}$, and $H_{2}$ along $e$ coincide. Hence, $e$ is degenerate.

At a triple point of a surface diagram $D$, there exist broken sheets $J_{1}, J_{2}, J_{3} \in$ $\mathcal{B}(D)$ uniquely (some of which possibly coincide) such that

- $J_{3}$ is the top sheet,

- $J_{2}$ is the middle sheet such that it is in back of $J_{3}$ with respect to the orientation of $J_{3}$, and

- $J_{1}$ is the bottom sheet such that it is in back of both $J_{2}$ and $J_{3}$ with respect to their orientations.

See the right of Figure 7. Note that we do not use the orientation of a bottom sheet for the definition of $J_{1}, J_{2}$ and $J_{3}$.

Definition 3.3. In the notation above, the color of each triple point is the triplet $\left(C\left(J_{1}\right), C\left(J_{2}\right), C\left(J_{3}\right)\right) \in R_{3} \times R_{3} \times R_{3}$. A triple point is of type $(n)$ for $n=1, \ldots, 5$ if the color satisfies the condition $(n)$ as listed in the following:

(1) $C\left(J_{1}\right)=C\left(J_{2}\right)=C\left(J_{3}\right)$.

(2) $C\left(J_{1}\right)=C\left(J_{2}\right) \neq C\left(J_{3}\right)$.

(3) $C\left(J_{1}\right) \neq C\left(J_{2}\right)=C\left(J_{3}\right)$.

(4) $C\left(J_{1}\right)=C\left(J_{3}\right) \neq C\left(J_{2}\right)$.

(5) $\left\{C\left(J_{1}\right), C\left(J_{2}\right), C\left(J_{3}\right)\right\}=\{0,1,2\}$.

A triple point of type (1)-(3) is called degenerate, and the others are called nondegenerate.

If we reverse the orientation of a tricolored surface diagram, then the type of each triple point changes such as $(n) \leftrightarrow(n)$ for $n=1,2,3$ and $(4) \leftrightarrow(5)$. Hence, the degeneracy does not change.

Let $\tau$ be a triple point of a tricolored surface diagram. If the color of $\tau$ is $(x, y, z) \in R_{3} \times R_{3} \times R_{3}$, then the broken sheets at $\tau$ are colored as shown in the left of Figure 8. Hence, the colors of the $\mathrm{b} / \mathrm{m}$-edges are $(x, y)$ and $(x * z, y * z)$, those of the $\mathrm{b} / \mathrm{t}$-edges are $(x, z)$ and $(x * y, z)$, and those of the $\mathrm{m} / \mathrm{t}$-edges are $(y, z)$ both. See the right of Figure 8, where the thin solid line, thick solid line, and dotted line show the $\mathrm{b} / \mathrm{m}-, \mathrm{b} / \mathrm{t}-$, and $\mathrm{m} / \mathrm{t}$-edges at $\tau$ respectively.

We denote by $d(\tau)$ the number of degenerate edges among six edges at $\tau$. Table 1 shows the colors of six edges at $\tau$ and $d(\tau)$ with respect to the color of $\tau$, where $\{a, b, c\}=\{0,1,2\}$. For example, if $(x, y, z)=(a, b, a)$, then $(x, y)=(a, b)$ and $(x * z, y * z)=(a, c)$ for the b/m-edges, $(x, z)=(a, a)$ and $(x * y, z)=(c, a)$ for the 


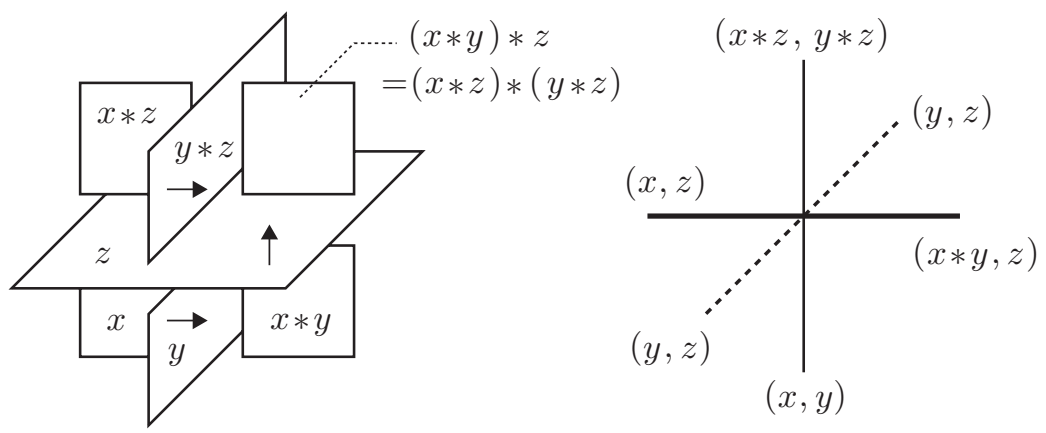

FIGURE 8.

$\mathrm{b} / \mathrm{t}$-edges, and $(y, z)=(b, a)$ for the $\mathrm{m} / \mathrm{t}$-edges both. Hence, we have $d(\tau)=1$ in this case. By Table 1, we see that $\tau$ is nondegenerate if and only if $d(\tau)=1$.

TABLE 1.

\begin{tabular}{|c|c|c|c|c|c|}
\hline \multirow{2}{*}{$\begin{array}{l}\text { type } \\
\text { of } \tau\end{array}$} & \multirow{2}{*}{$\begin{array}{c}\text { color } \\
\text { of } \tau\end{array}$} & \multicolumn{3}{|c|}{ colors of edges at $\tau$} & \multirow{2}{*}{$d(\tau)$} \\
\hline & & $\mathrm{b} / \mathrm{m}$-edges & b/t-edges & m/t-edges & \\
\hline (1) & $(a, a, a)$ & $(a, a),(a, a)$ & $(a, a),(a, a)$ & $(a, a),(a, a)$ & 6 \\
\hline$(2)$ & $\overline{(a, a, b)}$ & $(a, a),(c, c)$ & $(a, b),(a, b)$ & $(a, b),(a, b)$ & 2 \\
\hline$(3)$ & $(a, b, b)$ & $(a, b),(c, b)$ & $(a, b),(c, b)$ & $(b, b),(b, b)$ & 2 \\
\hline (4) & $\overline{(\overline{(a, b, a)}}$ & 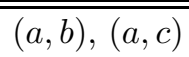 & (ב(a,a),(c,a) & ("b,a),(b,a) & 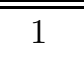 \\
\hline (5) & $(a, b, c)$ & $(a, b),(b, a)$ & $(a, c),(c, c)$ & $(b, c),(b, c)$ & 1 \\
\hline
\end{tabular}

At a triple point $\tau$ of an oriented surface diagram, let $\vec{n}_{1}, \vec{n}_{2}$, and $\vec{n}_{3}$ be the normal vectors to the bottom, middle, and top sheets respectively. The sign of $\tau$ is positive if the ordered triplet $\left(\vec{n}_{1}, \vec{n}_{2}, \vec{n}_{3}\right)$ is right-handed, and otherwise negative [9]. We denote by $\varepsilon(\tau) \in\{ \pm 1\}$ the sign of $\tau$. Let $\tau$ and $\tau^{\prime}$ be two triple points of a tricolored surface diagram. The pair $\left\{\tau, \tau^{\prime}\right\}$ is called a canceling pair if $\tau$ and $\tau^{\prime}$ have the same color and opposite signs.

Lemma 3.4. Let $\tau$ and $\tau^{\prime}$ be two triple points of a tricolored surface diagram which are nondegenerate with the same color. Assume that there is an edge e such that

(i) the endpoints of e are $\tau$ and $\tau^{\prime}$, and

(ii) $e$ is the nondegenerate $b / t$-edge at both $\tau$ and $\tau^{\prime}$.

Then we have $\varepsilon(\tau)=-\varepsilon\left(\tau^{\prime}\right)$, and hence, $\left\{\tau, \tau^{\prime}\right\}$ is a canceling pair.

Proof. By reversing the orientation of the surface diagram if necessary, we may assume that both $\tau$ and $\tau^{\prime}$ are of type (4) with the same color $(a, b, a)$, and that the edge $e$ satisfies the conditions (i) and (ii) in the lemma. Consider a neighborhood of the edge $e$. Since the color of $e$ is $(c, a)$ by Table 1, the three broken sheets around $e$ are tricolored as in Figure 9(i), where $\{a, b, c\}=\{0,1,2\}$. Since the other $\mathrm{b} / \mathrm{t}$-edges at $\tau$ and $\tau^{\prime}$ have the color $(a, a)$, all the sheets around $\tau$ and $\tau^{\prime}$ must be tricolored as in Figure 9(ii). Since the color of $\tau$ and $\tau^{\prime}$ is $(a, b, a)$, the orientations of the middle sheets are opposite as in Figure 9(iii). Hence, we have $\varepsilon(\tau)=-\varepsilon\left(\tau^{\prime}\right)$. 


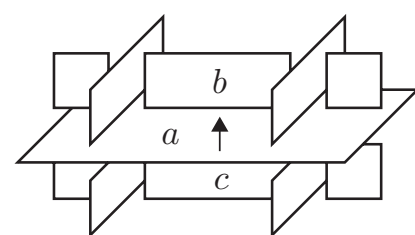

(i)

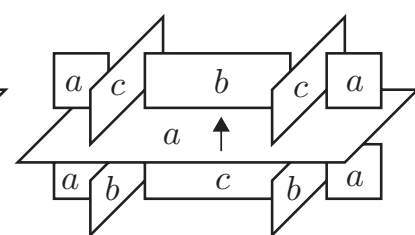

(ii)

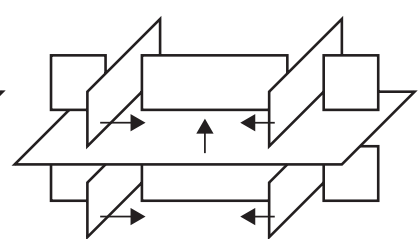

(iii)

FIGURE 9.

\section{Nondegenerate TRIPLE POINTS}

The following Propositions 4.1-4.3 concerning nondegenerate triple points are essential for the proof of Theorem 1.2.

Proposition 4.1. In any tricolored surface diagram, the number of nondegenerate triple points is even.

Proof. Let $B$ and $E_{0}$ be the numbers of branch points and degenerate edges of a tricolored surface diagram respectively, and $\left\{\tau_{1}, \ldots, \tau_{s}\right\}$ the set of triple points of the diagram. By counting the degenerate edges around branch points and triple points, we have

$$
2 E_{0}=B+\sum_{i=1}^{s} d\left(\tau_{i}\right)
$$

by Lemma 3.2. Since $B$ is even, we see that $\sum_{i=1}^{s} d\left(\tau_{i}\right)$ is even from the above equation. On the other hand, since $d(\tau)=1,2,6$ by Table $1, \sum_{i=1}^{s} d\left(\tau_{i}\right)$ has the same parity as the number of triple points with $d\left(\tau_{i}\right)=1$. Since $d\left(\tau_{i}\right)=1$ if and only if $\tau_{i}$ is nondegenerate, we have the conclusion.

Proposition 4.2. Assume that a tricolored surface diagram has exactly two triple points $\tau$ and $\tau^{\prime}$. If both $\tau$ and $\tau^{\prime}$ are nondegenerate, then $\left\{\tau, \tau^{\prime}\right\}$ is a canceling pair.

Proof. We consider the following three cases with respect to the types of $\tau$ and $\tau^{\prime}$.

Case 1. Assume that $\tau$ is of type (4) with the color $(a, b, a)$ and that $\tau^{\prime}$ is of type (5) with the color $\left(a^{\prime}, b^{\prime}, c^{\prime}\right)$, where $\{a, b, c\}=\left\{a^{\prime}, b^{\prime}, c^{\prime}\right\}=\{0,1,2\}$. Let $e_{1}$ and $e_{2}$ be the $\mathrm{m} / \mathrm{t}$-edges at $\tau$, whose colors are $(b, a)$ by Table 1 . By Lemmas 2.3 and 3.2, another endpoint of each $e_{i}$ is $\tau^{\prime}$. [This can be seen as follows. Since $e_{1}$ is nondegenerate, another endpoint of $e_{1}$ is not a branch point by Lemma 3.2. If both endpoints of $e_{1}$ are $\tau$, then $e_{1}$ and $e_{2}$ coincide because there are no edges at $\tau$ with the color $(b, a)$ except $e_{1}$ and $e_{2}$. Hence, we have the cycle $e_{1}=e_{2}$ with the single triple point $\tau$ on it. This contradicts Lemma 2.3.] On the other hand, there is no pair of edges at $\tau^{\prime}$ with the same color, except the $\mathrm{m} / \mathrm{t}$-edges, whose color is $\left(b^{\prime}, c^{\prime}\right)$. Hence, we have $(b, a)=\left(b^{\prime}, c^{\prime}\right)$, and the color of $\tau^{\prime}$ is $(c, b, a)$. In Table 2 , we list the colors of edges at $\tau$ and $\tau^{\prime}$. Let $e_{3}$ be the $\mathrm{b} / \mathrm{m}$-edge at $\tau$ with the color $(a, b)$. By observing Table 2 and using Lemma 3.2, we conclude that another endpoint of $e_{3}$ is neither $\tau, \tau^{\prime}$ nor a branch point. Hence, this case cannot happen.

Case 2. Assume that both $\tau$ and $\tau^{\prime}$ are of type (4) with the colors $(a, b, a)$ and $\left(a^{\prime}, b^{\prime}, a^{\prime}\right)$ respectively, where $\{a, b, c\}=\left\{a^{\prime}, b^{\prime}, c^{\prime}\right\}=\{0,1,2\}$. It follows from an argument similar to Case 1 that $(a, b, c)=\left(a^{\prime}, b^{\prime}, c^{\prime}\right)$ and that each nondegenerate edge connects $\tau$ and $\tau^{\prime}$. Since the edge with the color $(c, a)$ satisfies the condition in Lemma 3.4, $\left\{\tau, \tau^{\prime}\right\}$ is a canceling pair. 
TABLE 2 .

\begin{tabular}{|c|c||c|c|c|}
\hline & color & $\mathrm{b} /$ m-edges & $\mathrm{b} /$ t-edges & $\mathrm{m} /$ t-edges \\
\hline \hline$\tau$ & $(a, b, a)$ & $(a, b),(a, c)$ & $(a, a),(c, a)$ & $(b, a),(b, a)$ \\
\hline$\tau^{\prime}$ & $(c, b, a)$ & $(c, b),(b, c)$ & $(c, a),(a, a)$ & $(b, a),(b, a)$ \\
\hline
\end{tabular}

Case 3. Assume that both $\tau$ and $\tau^{\prime}$ are of type (5). Consider the tricolored surface diagram obtained by reversing the orientation of the original one, in which $\tau$ and $\tau^{\prime}$ are of type (4). By Case 2, $\left\{\tau, \tau^{\prime}\right\}$ is a canceling pair in the orientation-reversed diagram, and hence, it is also a canceling pair of the original one.

Proposition 4.3. Assume that a tricolored surface diagram has exactly three triple points $\tau, \tau^{\prime}$, and $\tau^{\prime \prime}$. If $\tau$ and $\tau^{\prime}$ are nondegenerate and $\tau^{\prime \prime}$ is degenerate, then $\left\{\tau, \tau^{\prime}\right\}$ is a canceling pair.

Proof. We consider the following three cases with respect to the type of $\tau^{\prime \prime}$.

Case 1. Assume that $\tau^{\prime \prime}$ is of type (1). Since all six edges at $\tau^{\prime \prime}$ are degenerate, this case can be proved in a similar way to Proposition 4.2.

Case 2. Assume that $\tau^{\prime \prime}$ is of type (2). Among six edges at $\tau^{\prime \prime}$, there are two degenerate edges, say $e_{1}$ and $e_{2}$, which are $\mathrm{b} / \mathrm{m}$-edges at $\tau^{\prime \prime}$. See Table 1 . First, consider the case that at least one of $e_{1}$ and $e_{2}$ connects to a branch point. Since the edge is a $\mathrm{b} / \mathrm{m}$-edge at $\tau^{\prime \prime}$, we can reduce this case to Proposition 4.2 by using Lemma 2.2 so that $\left\{\tau, \tau^{\prime}\right\}$ is a canceling pair. Next, consider the case that another endpoint of each $e_{i}(i=1,2)$ is a triple point. However, this case cannot happen; since $e_{1}$ and $e_{2}$ are distinct by Lemma 2.3, another endpoint of each $e_{i}$ is $\tau$ or $\tau^{\prime}$. On the other hand, since $\tau$ and $\tau^{\prime}$ are nondegenerate, they have exactly one degenerate edge each. Hence, we may assume that $e_{1}$ connects $\tau^{\prime \prime}$ and $\tau$, and that $e_{2}$ connects $\tau^{\prime \prime}$ and $\tau^{\prime}$. It follows from Lemma 3.2 that there is no edge of the surface diagram whose endpoints are a branch point and a triple point. This contradicts Lemma 2.1 .

Case 3. Assume that $\tau^{\prime \prime}$ is of type (3). Consider the tricolored surface diagram obtained by reversing the orientation of the original one, in which $\tau^{\prime \prime}$ is of type (2). By Case $2,\left\{\tau, \tau^{\prime}\right\}$ is a canceling pair of the orientation-reversed diagram, and hence, it is also a canceling pair of the original one.

\section{Cocycle invariants OF SURFACE-KNOtS}

Let $D$ be a surface diagram of an oriented surface-knot $F$ whose triple points are $\tau_{1}, \ldots, \tau_{s}$ with the signs $\varepsilon_{i}=\varepsilon\left(\tau_{i}\right)$. For a tricoloring $C$ for $D$, let $\left(a_{i}, b_{i}, c_{i}\right) \in$ $R_{3} \times R_{3} \times R_{3}$ be the color of $\tau_{i}(i=1, \ldots, s)$. For an Abelian group $G$ in which the sum is written multiplicatively, we consider a map $\theta: R_{3} \times R_{3} \times R_{3} \rightarrow G$. Then we put

$$
W_{\theta}\left(\tau_{i} ; C\right)=\theta\left(a_{i}, b_{i}, c_{i}\right)^{\varepsilon_{i}} \in G
$$

for each triple point $\tau_{i}$, and

$$
W_{\theta}(C)=\prod_{i=1}^{s} W_{\theta}\left(\tau_{i} ; C\right) \in G
$$


for the tricoloring $C$. Since the set of broken sheets of $D$ is finite, so is the set of tricolorings for $D$. Let $C_{1}, \ldots, C_{n}$ be the tricolorings for $D$. Then we put

$$
\Phi_{\theta}(D)=\sum_{j=1}^{n} W_{\theta}\left(C_{j}\right) \in \mathbb{Z}[G] .
$$

Note that if $\Phi_{\theta}(D) \notin \mathbb{Z} \subset \mathbb{Z}[G]$, then there is a tricoloring $C$ with $W_{\theta}(C) \neq 1_{G} \in G$ by definition. The following theorem is proved in [3], where $\Phi_{\theta}(D)$ is defined not only for $R_{3}$ but for any finite quandle.

Theorem 5.1 ([3]). In the notation above, if a map $\theta: R_{3} \times R_{3} \times R_{3} \rightarrow G$ satisfies

(i) $\theta(x, y, z)=1_{G}$ for $x=y$ or $y=z$, and

(ii) for any $x, y, z, w \in R_{3}$,

$$
\begin{aligned}
\theta(x, z, w) & \cdot \theta(x, y, w)^{-1} \cdot \theta(x, y, z) \\
& =\theta(x * y, z, w) \cdot \theta(x * z, y * z, w)^{-1} \cdot \theta(x * w, y * w, z * w),
\end{aligned}
$$

then $\Phi_{\theta}(D)$ is independent of the choice of a surface diagram $D$ of $F$.

When a map $\theta$ satisfies the conditions (i) and (ii) in Theorem 5.1, $\Phi_{\theta}(D)$ is called a cocycle invariant of $F$ with respect to the 3-cocycle $\theta$, and denoted by $\Phi_{\theta}(F)$. Refer to [2, 4] also.

Example 5.2. In 24], Mochizuki finds an explicit presentation of a 3-cocycle $\theta: R_{3} \times R_{3} \times R_{3} \rightarrow \mathbb{Z}_{3}=\left\langle t \mid t^{3}=1\right\rangle$ such that

$$
\theta(x, y, z)=t^{(x-y)(y-z) z(x+z)} \in \mathbb{Z}_{3} .
$$

The reader can check that this map $\theta$ satisfies the conditions (i) and (ii) in Theorem 5.1 by hand.

Proof of Theorem 1.2. From the definition of $\Phi_{\theta}(F)$, it is sufficient to prove that if a surface diagram $D$ of $F$ has at most three triple points, then we have $W_{\theta}(C)=$ $1_{G} \in G$ for any 3 -cocycle $\theta$ and any tricoloring $C$ for $D$. By Proposition 4.1, the number of nondegenerate triple points is zero or two. So there are three cases to consider.

Case 1. Assume that all the triple points of $D$ are degenerate. By condition (i) in Theorem 5.1, we have $W_{\theta}(\tau ; C)=1_{G}$ for any triple point $\tau$ of $D$, and hence, $W_{\theta}(C)=\prod_{\tau} W_{\theta}(\tau ; C)=1_{G}$.

Case 2. Assume that the surface diagram $D$ has exactly two triple points, say $\tau$ and $\tau^{\prime}$, such that they are nondegenerate. By Proposition 4.2 , we have $W_{\theta}(\tau ; C)=$ $W_{\theta}\left(\tau^{\prime} ; C\right)^{-1}$, and hence, $W_{\theta}(C)=W_{\theta}(\tau ; C) \cdot W_{\theta}\left(\tau^{\prime} ; C\right)=1_{G}$.

Case 3. Assume that the surface diagram $D$ has exactly three triple points, say $\tau, \tau^{\prime}$, and $\tau^{\prime \prime}$, such that $\tau$ and $\tau^{\prime}$ are nondegenerate and $\tau^{\prime \prime}$ is degenerate. By Proposition 4.3, we have $W_{\theta}(\tau ; C)=W_{\theta}\left(\tau^{\prime} ; C\right)^{-1}$ and $W_{\theta}\left(\tau^{\prime \prime} ; C\right)=1_{G}$, and hence, $W_{\theta}(C)=W_{\theta}(\tau ; C) \cdot W_{\theta}\left(\tau^{\prime} ; C\right) \cdot W_{\theta}\left(\tau^{\prime \prime} ; C\right)=1_{G}$.

\section{Motion PICTURES}

A motion of a tangle $T \subset \mathbb{R}_{+}^{3}$ is a continuous series of tangles $M=\left\{T_{t}\right\}_{t \in I}$ such that $T_{0}=T_{1}=T$ and $\partial T_{t}=\partial T$ for any $t \in I=[0,1]$. We associate $M$ with a 2-disk $\Delta_{M}$ embedded in $\mathbb{R}_{+}^{3} \times I$ as follows:

$$
\Delta_{M}=\bigcup_{t \in I} T_{t} \times\{t\} \subset \mathbb{R}_{+}^{3} \times I .
$$


Two motions $M$ and $M^{\prime}$ are equivalent if there is an ambient isotopy $\left\{h_{s}\right\}_{s \in I}$ of $\mathbb{R}_{+}^{3} \times I$ which maps $\Delta_{M}$ onto $\Delta_{M^{\prime}}$ such that $h_{s}$ is the identity on $\partial \mathbb{R}_{+}^{3} \times I$ and $h_{s} \mid \mathbb{R}_{+}^{3} \times\{0\}$ agrees with $h_{s} \mid \mathbb{R}_{+}^{3} \times\{1\}$ for any $s \in I$.

Let $\pi^{\prime}: \mathbb{R}_{+}^{3} \rightarrow \mathbb{R}_{+}^{2}$ be a projection. Under the projection $\pi^{\prime} \times \operatorname{id}_{I}: \mathbb{R}_{+}^{3} \times I \rightarrow$ $\mathbb{R}_{+}^{2} \times I$, the image of $\Delta_{M}$ is assumed to be a generic 2-disk in $\mathbb{R}_{+}^{2} \times I$. We denote by $\Delta_{M}^{*}$ the projection image with crossing information which is a surface diagram of $\Delta_{M}$. Then $\Delta_{M}^{*}$ is regarded as a continuous series of tangle diagrams $\left\{T_{t}^{*}\right\}_{t \in I}$, where $T_{t}^{*}$ is a tangle diagram $\pi^{\prime}\left(T_{t}\right)$ with crossing information:

$$
\Delta_{M}^{*}=\bigcup_{t \in I} T_{t}^{*} \times\{t\} \subset \mathbb{R}_{+}^{2} \times I .
$$

The series $\left\{T_{t}^{*}\right\}_{t \in I}$ of tangle diagrams is called the motion picture of a motion $M$, and is denoted by $P_{M}$. Such a motion picture contains a finite number of Reidemeister moves on tangle diagrams. Then we have the following. See Figure 10 .

Lemma 6.1 (cf. 3] 31]). A Reidemeister move I or III in a motion picture $P_{M}=\left\{T_{t}^{*}\right\}_{t \in I}$ corresponds to a branch point or a triple point of the surface diagram $\Delta_{M}^{*} \subset \mathbb{R}_{+}^{2} \times I$ respectively.
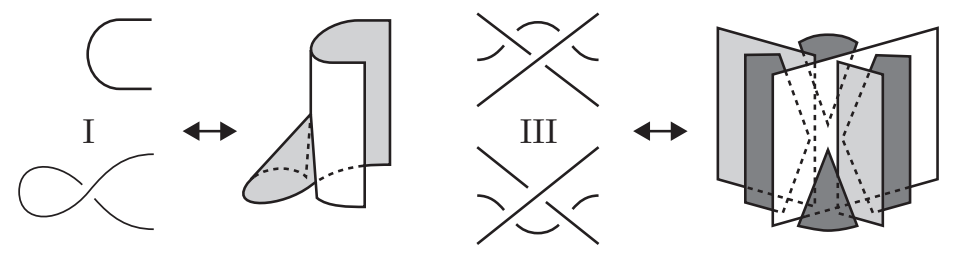

FiguRE 10.

We remark that a Reidemeister move II in a motion picture $P_{M}$ corresponds to a maximal or minimal point of the singularity set of $\Delta_{M}^{*}$ with respect to the height function $\mathbb{R}_{+}^{2} \times I \rightarrow I$.

Example 6.2 (31]). Consider a motion $M$ of a tangle $T$ as shown in Figure $11 \mathrm{a} \rightarrow \mathrm{f}$. The deformations from one to the next are indicated by the arrows, and the boxed sub-tangle does not deformed during the moves. Then $M$ is equivalent to the motion corresponding to one twist of $T$. As depicted in the figure, we take a motion picture $P_{M}$ by the projection $\pi^{\prime}: \mathbb{R}_{+}^{3} \rightarrow \mathbb{R}_{+}^{2}$ whose direction is normal to the paper. In the process of $\mathrm{a} \rightarrow \mathrm{b}$ (or $\mathrm{c} \rightarrow \mathrm{d}$ ), the boxed diagram goes over (or under) a root-arc of the tangle. If the box contains $c$ crossings, then there are $c$ Reidemeister move III's and some II's in each process. Moreover, the process $\mathrm{b} \rightarrow \mathrm{c}$ (or $\mathrm{e} \rightarrow \mathrm{f}$ ) is made by a Reidemeister move I, and $\mathrm{d} \rightarrow \mathrm{e}$ is by a move II. Hence, $P_{M}$ has $2 c$ Reidemeister move III's, and the corresponding surface diagram $\Delta_{M}^{*} \subset \mathbb{R}_{+}^{2} \times I$ has also $2 c$ triple points by Lemma 6.1.

To obtain $\mathbb{R}^{4}$ from $\mathbb{R}_{+}^{3} \times I$, we consider the identification on $\mathbb{R}_{+}^{3} \times I$ such that

- $(x, t)=\left(x, t^{\prime}\right)$ for any $x \in \partial \mathbb{R}_{+}^{3}$ and $t, t^{\prime} \in I$, and

- $(x, 0)=(x, 1)$ for any $x \in \mathbb{R}_{+}^{3}$. 


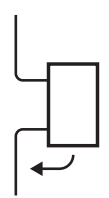

a

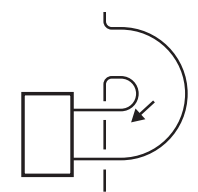

b

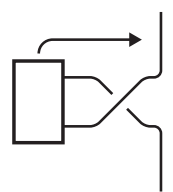

c

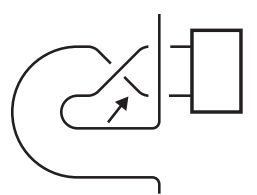

d

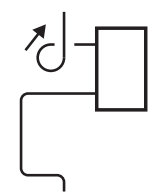

e

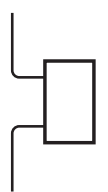

$\mathrm{f}$

Figure 11.

Let $q: \mathbb{R}_{+}^{3} \times I \rightarrow \mathbb{R}^{4}$ denote this quotient map. The deform-spin associated with a motion $M$ is a 2 -knot $q\left(\Delta_{M}\right) \subset \mathbb{R}^{4}$, and is denoted by $F_{M}[20$. We remark that if two motions $M$ and $M^{\prime}$ are equivalent, then the deform-spins $F_{M}$ and $F_{M^{\prime}}$ are also equivalent.

Similarly, let $q^{\prime}: \mathbb{R}_{+}^{2} \times I \rightarrow \mathbb{R}^{3}$ be the quotient map with the identification of $\partial \mathbb{R}_{+}^{2} \times I$ with $\partial \mathbb{R}_{+}^{2}$ and $\mathbb{R}_{+}^{2} \times\{0\}$ with $\mathbb{R}_{+}^{2} \times\{1\}$. Since the projection $\pi^{\prime} \times \mathrm{id}_{I}: \mathbb{R}_{+}^{3} \times$ $I \rightarrow \mathbb{R}_{+}^{2} \times I$ induces a projection $\pi: \mathbb{R}^{4} \rightarrow \mathbb{R}^{3}$ naturally, we see that $q^{\prime}\left(\Delta_{M}^{*}\right) \subset \mathbb{R}^{3}$ is a surface diagram of $F_{M}$ denoted by $D_{M}$ :

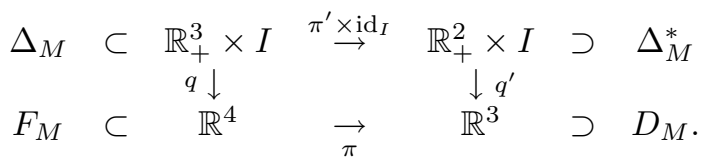

For a motion $M$, we denote by $M^{m}$ the motion obtained by repeating $M m$ times $\left(m \geq 0\right.$; in case of $m=0$, put $T_{t}=T_{0}$ for any $\left.t \in I\right)$. Let $T$ be a tangle in $\mathbb{R}_{+}^{3}$ whose knotting is a classical knot $K$. Let $M_{T}$ be a motion which presents one twist of $T$. Then the deform-spin associated with the motion $\left(M_{T}\right)^{m}$ is called the $m$ twist-spin of $K$ and denoted by $\tau^{m} K$ [35]. Assume that $K$ has a knot diagram with $c$ crossings. Then there is a tangle diagram of $T$ having $c$ crossings. By Example 6.2 , the $m$-twist-spin of $K, \tau^{m} K=F_{\left(M_{T}\right)^{m}}$, has a surface diagram $D_{\left(M_{T}\right)^{m}}$ with $2 c \cdot m$ triple points; that is, $\mathrm{t}\left(\tau^{m} K\right) \leq 2 \mathrm{~cm}$. In [31, we improve this estimation so that we have $\mathrm{t}\left(\tau^{m} K\right) \leq 2(c-1) m$ for $c>0$.

Proof of Theorem 1.3. Let $T$ be a tangle whose knotting is $K$. Note that $T$ has a tangle diagram as in the top row of Figure 12 by assumption. Consider two motions $M$ and $M^{\prime}$ of $T$ as shown in columns (i) and (iii) of the figure respectively. Then there is a 3 -ball $B^{3}$ in $\mathbb{R}_{+}^{3} \times I$ such that

- the closure of $\left(\Delta_{M} \cup \Delta_{M^{\prime}}\right) \backslash\left(\Delta_{M} \cap \Delta_{M^{\prime}}\right)$ is the boundary $\partial B^{3}$,

- $\Delta_{M} \cap B^{3}=\Delta_{M} \cap \partial B^{3}$ is a 2-disk, and

- $\Delta_{M^{\prime}} \cap B^{3}=\Delta_{M^{\prime}} \cap \partial B^{3}$ is also a 2-disk.

In column (ii) of Figure 12, we describe each slice $B^{3} \cap \mathbb{R}_{+}^{3} \times\{t\}$ by a shaded 2-disk. By the cellular move lemma in [18. p. 84, Proposition 1.7] or [26, p. 55], there is an ambient isotopy of $\mathbb{R}_{+}^{3} \times I$ mapping $\Delta_{M}$ onto $\Delta_{M^{\prime}}$ and keeping $\mathbb{R}_{+}^{3} \times I \backslash N\left(B^{3}\right)$ fixed, where $N\left(B^{3}\right)$ is a regular neighborhood of $B^{3}$ in $\mathbb{R}_{+}^{3} \times I$. Hence, $M^{\prime}$ is equivalent to $M$. Since the motion $M$ is the same as that given in Example 6.2, $M^{\prime}$ is equivalent to the motion $M_{T}$ presenting one twist of $T$.

As depicted in the figure, we take a motion picture $P_{M^{\prime}}$ of $M^{\prime}$ by the projection $\pi^{\prime}: \mathbb{R}_{+}^{3} \rightarrow \mathbb{R}_{+}^{2}$ whose direction is normal to the paper. By the assumption of the theorem, we may assume that the boxed subdiagram in $P_{M^{\prime}}$ has $c-2$ crossings. 
In the process of $\mathrm{a} \rightarrow \mathrm{b}$ ( or $\mathrm{c} \rightarrow \mathrm{d}$ ), only the boxed part goes over (or under) a rootarc without passing two crossings adjacent to the box. Hence, there are $c-2$ Reidemeister move III's in each of the processes $\mathrm{a} \rightarrow \mathrm{b}$ and $\mathrm{c} \rightarrow \mathrm{d}$. Moreover, the process $\mathrm{b} \rightarrow \mathrm{c}$ is made by a Reidemeister move II and a move $\mathrm{I}, \mathrm{d} \rightarrow \mathrm{e}$ is by two move II's, and $\mathrm{e} \rightarrow \mathrm{f}$ by a move I. Thus we see that $P_{M^{\prime}}$ has $2(c-2)$ Reidemeister move III's, so that the surface diagram $\Delta_{M^{\prime}}^{*} \subset \mathbb{R}_{+}^{2} \times I$ has $2(c-2)$ triple points. Hence, the $m$-twist-spin $\tau^{m} K$ has a surface diagram $D_{\left(M^{\prime}\right)^{m}}$ with $2(c-2) m$ triple points.

\section{Demonstration for the 2-TWIST-SPUn TREFOIL}

In this section, we demonstrate that the 2-twist-spun trefoil $\tau^{2}$ (trefoil) has the cocycle invariant $\Phi_{\theta}\left(\tau^{2}\right.$ (trefoil $\left.)\right)=3+6 t^{2} \in \Lambda_{3}=\mathbb{Z}\left[t, t^{-1}\right] /\left(t^{3}-1\right)$ with respect to Mochizuki's 3-cocycle $\theta$ given in Example 5.2. The calculation is divided into several steps in which we use the notation of the previous sections.

1. Surface diagram. Let $T$ be a tangle whose knotting is the trefoi, and $T^{*}$ a tangle diagram of $T$ as shown in the top (or bottom) right of Figure 13, where we label the crossings by $X, Y$ and $Z$ (or $X^{\prime}, Y^{\prime}$ and $Z^{\prime}$ ). Consider the motion $M^{\prime}$ and its motion picture $P_{M^{\prime}}=\left\{T_{t}^{*}\right\}_{t \in I}$ given in the proof of Theorem 1.3 (see column (iii) of Figure 12) with $T_{0}^{*}=T_{1}^{*}=T^{*}$. Then a regular neighborhood of the singularity set of the surface diagram $\Delta_{M^{\prime}}^{*}$ in $\mathbb{R}_{+}^{2} \times I$ is as shown in the left of Figure 13. We denote it by $\Gamma$. More precisely, we take a neighborhood $\Gamma_{t}$ of crossings of $T_{t}^{*}$ in $\mathbb{R}_{+}^{2} \times\{t\}$ so that we obtain $\Gamma=\bigcup_{t \in I} \Gamma_{t} \times\{t\}$. In particular, the crossings $X, Y, Z$ of $T_{0}^{*}$ (or $X^{\prime}, Y^{\prime}, Z^{\prime}$ of $T_{1}^{*}$ ) correspond to the top (or bottom) end-parts of $\Gamma$ with the same labels.

Take two copies $\Gamma_{1}$ and $\Gamma_{2}$ of $\Gamma$. Then the singularity set of the surface diagram $D_{\left(M^{\prime}\right)^{2}}$ of $F_{\left(M^{\prime}\right)^{2}}=\tau^{2}$ (trefoil) has a regular neighborhood $\Gamma_{1} \cup \Gamma_{2}$ by connecting $X^{\prime}, Y^{\prime}, Z^{\prime}$ of $\Gamma_{1}$ with $X, Y, Z$ of $\Gamma_{2}$ respectively and vice versa like a closure of a braid. It is not difficult to see that $D_{\left(M^{\prime}\right)^{2}} \backslash\left(\Gamma_{1} \cup \Gamma_{2}\right)$ is a disjoint union of fourteen open 2-disks. Hence, the surface diagram $D_{\left(M^{\prime}\right)^{2}}$ is constructed from $\Gamma_{1} \cup \Gamma_{2}$ by gluing a 2-disk along each component of the boundary of $\Gamma_{1} \cup \Gamma_{2}$ without making any singularities.

2. Signs of triple points. The motion picture $P_{M^{\prime}}$ has two Reidemeister move III's and two I's. Hence, there are two triple points and two branch points in $\Gamma \subset \Delta_{M^{\prime}}^{*}$ by Lemma 6.1. We denote by $\tau_{1}$ and $\tau_{2}$ (or $\tau_{3}$ and $\tau_{4}$ ) the corresponding triple points of $\Gamma_{1}$ (or $\Gamma_{2}$ ) as shown in Figure 13. We fix an orientation of $\Gamma$ (and hence, $\Gamma_{1}$ and $\Gamma_{2}$ ) as indicated by the arrows in the figure. Then the signs of the $\tau_{i}$ 's are $\varepsilon\left(\tau_{1}\right)=\varepsilon\left(\tau_{3}\right)=+1$ and $\varepsilon\left(\tau_{2}\right)=\varepsilon\left(\tau_{4}\right)=-1$. Note that the orientation of $\Gamma$ extends to that of the surface diagram $D_{\left(M^{\prime}\right)^{2}}$ naturally.

3. Tricolorings. The surface diagram $D_{\left(M^{\prime}\right)^{2}}$ admits three trivial tricolorings, that is, a single color is used for the coloring. We denote by $C_{a}$ the trivial tricoloring with a color $a \in\{0,1,2\}$. Consider nontrivial tricolorings for $D_{\left(M^{\prime}\right)^{2}}$. We see that the tangle diagram $T_{0}^{*}$ of the trefoil admits six nontrivial tricolorings, denoted by $C_{a b c}$, as shown in the top right of Figure 13, where $\{a, b, c\}=\{0,1,2\}$. The tricoloring $C_{a b c}$ for $T_{0}^{*}$ determines that for the top parts $X, Y$ and $Z$ of $\Gamma$, which extend to all of $\Gamma$ uniquely as shown in the left of the figure. Observe that the colors of the bottom end-parts $X^{\prime}, Y^{\prime}, Z^{\prime}$ of $\Gamma$ coincide with the top ones with $b$ and $c$ interchanged. Hence, by giving $C_{a b c}$ to $\Gamma_{1}$ and $C_{a c b}$ to $\Gamma_{2}$, we have a tricoloring for $\Gamma_{1} \cup \Gamma_{2}$. This tricoloring for $\Gamma_{1} \cup \Gamma_{2}$ is extended to the fourteen 


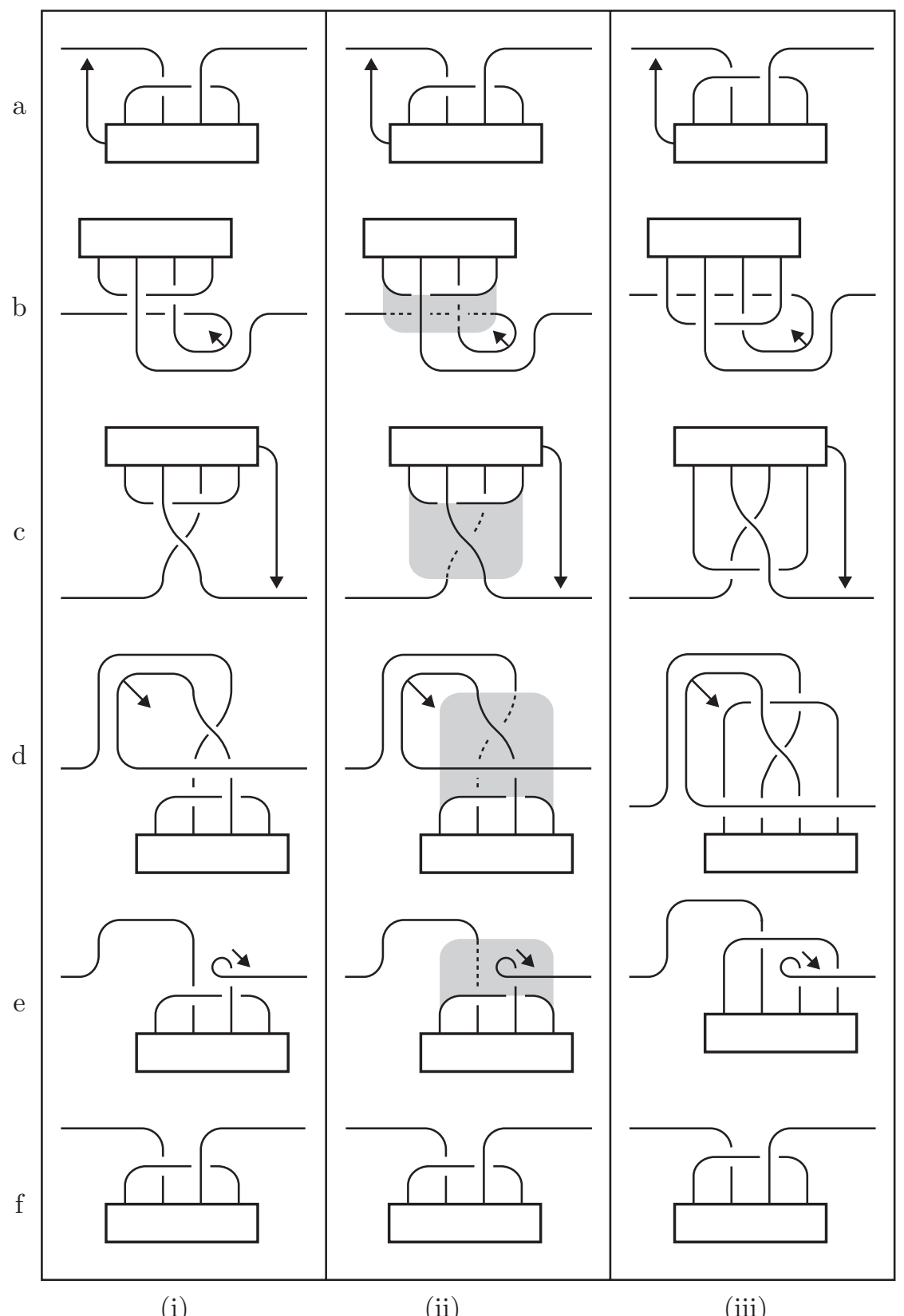

(i)

(ii)

(iii)

FiguRE 12.

disks of $D_{\left(M^{\prime}\right)^{2}} \backslash\left(\Gamma_{1} \cup \Gamma_{2}\right)$ so that we obtain a tricoloring for $D_{\left(M^{\prime}\right)^{2}}$. We denote this tricoloring for $D_{\left(M^{\prime}\right)^{2}}$ by $C_{a b c}$ also. Conversely, any nontrivial tricolorings for $D_{\left(M^{\prime}\right)^{2}}$ are obtained as above.

4. Colors of triple points. For a trivial tricoloring $C_{a}$ for $D_{\left(M^{\prime}\right)^{2}}$, all the colors of $\tau_{i}$ 's are $(a, a, a)$. On the other hand, for a nontrivial tricoloring $C_{a b c}$ for 


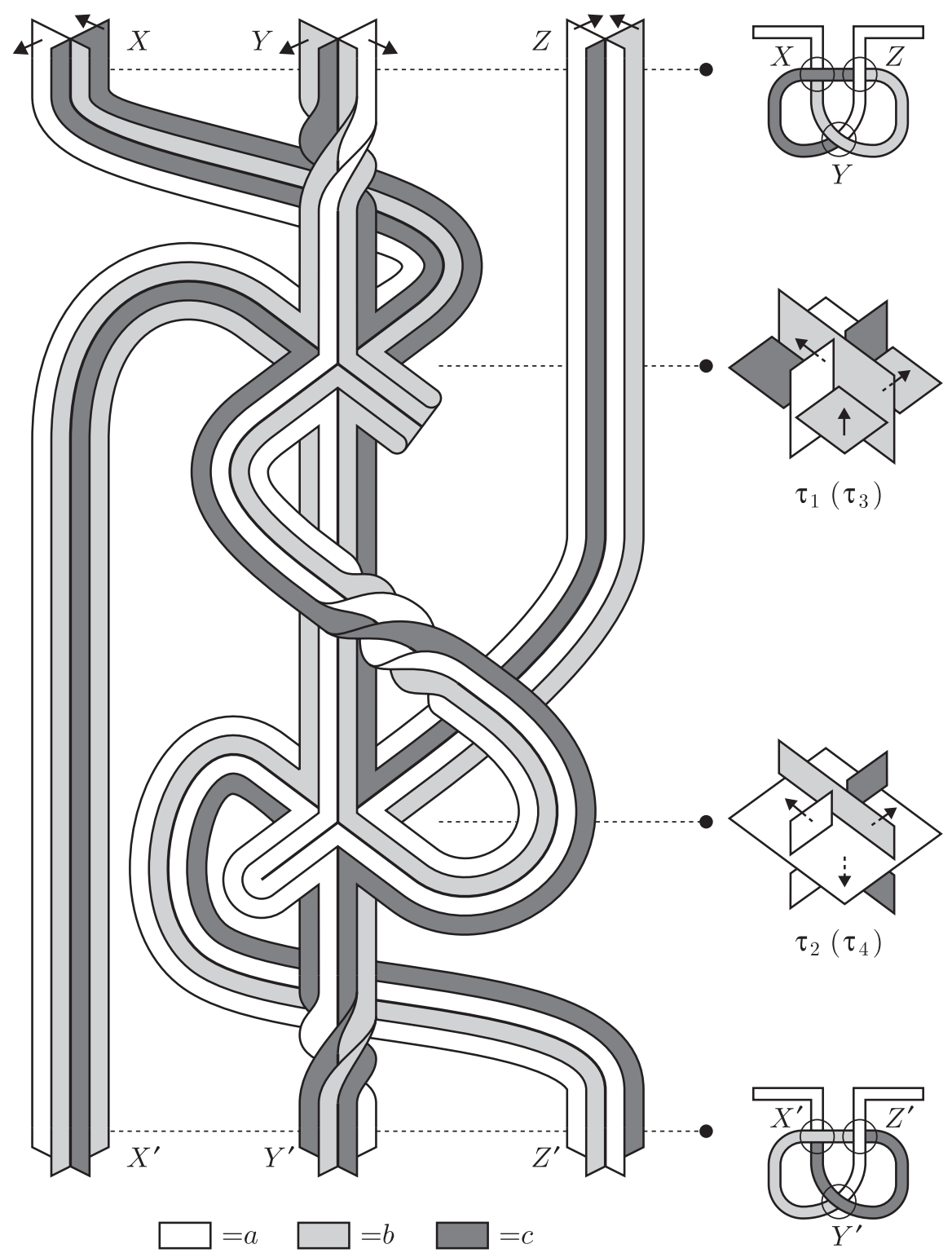

FigURE 13.

$D_{\left(M^{\prime}\right)^{2}}$, the triple points $\tau_{1}$ and $\tau_{2}$ have the colors $(b, a, b)$ and $(a, b, a)$, respectively, as shown in the right middle of Figure 13. By interchanging $b$ and $c$, we see that the colors of $\tau_{3}$ and $\tau_{4}$ are $(c, a, c)$ and $(a, c, a)$ respectively. We summarize the data for the calculation of a cocycle invariant as shown in Table 3.

5. Cocycle invariant. Let $\theta: R_{3} \times R_{3} \times R_{3} \rightarrow \mathbb{Z}_{3}=\left\langle t \mid t^{3}=1\right\rangle$ be Mochizuki's 3 -cocycle given in Example 5.2. First, we have $W_{\theta}\left(C_{a}\right)=1$ for each $a \in\{0,1,2\}$ by definition. On the other hand, since

$$
\theta(x, y, x)=t^{(x-y)(y-x) x(x+x)}=t^{x^{2}} \in \mathbb{Z}_{3}
$$


TABLE 3 .

\begin{tabular}{|c||c|c|c|c|}
\hline$\tau_{i}$ & $\tau_{1}$ & $\tau_{2}$ & $\tau_{3}$ & $\tau_{4}$ \\
\hline$\varepsilon\left(\tau_{i}\right)$ & + & - & + & - \\
\hline \hline$C_{a}$ & $(a, a, a)$ & $(a, a, a)$ & $(a, a, a)$ & $(a, a, a)$ \\
\hline$C_{a b c}$ & $(b, a, b)$ & $(a, b, a)$ & $(c, a, c)$ & $(a, c, a)$ \\
\hline
\end{tabular}

for any $x, y \in R_{3}$ with $x \neq y$, we have

$$
W_{\theta}\left(C_{a b c}\right)=\prod_{i=1}^{4} W_{\theta}\left(\tau_{i} ; C_{a b c}\right)=t^{b^{2}-a^{2}+c^{2}-a^{2}}=t^{a^{2}+b^{2}+c^{2}}=t^{2} \in \mathbb{Z}_{3}
$$

for each tricoloring $C_{a b c}$ with $\{a, b, c\}=\{0,1,2\}$. Hence, the cocycle invariant of the 2-twist-spun trefoil with respect to Mochizuki's 3-cocycle $\theta$ is

$$
\begin{aligned}
\Phi_{\theta}\left(\tau^{2}(\text { trefoil })\right) & =\sum_{a \in\{0,1,2\}} W_{\theta}\left(C_{a}\right)+\sum_{\{a, b, c\}=\{0,1,2\}} W_{\theta}\left(C_{a b c}\right) \\
& =3+6 t^{2} \in \Lambda_{3}=\mathbb{Z}\left[t, t^{-1}\right] /\left(t^{3}-1\right) .
\end{aligned}
$$

\section{REFERENCES}

[1] T. F. Banchoff, Double tangency theorems for pairs of submanifolds, in Geometry Symposium, Utrecht 1980 ed. Looijenga, Seirsma, and Takens, LNM v. 894, Springer-Verlag (1981), 26-48. MR 83h:53005

[2] J. S. Carter, D. Jelsovsky, S. Kamada, L. Langford and M. Saito, State-sum invariants of knotted curves and surfaces from quandle cohomology, Electron. Res. Announc. Amer. Math. Soc. 5 (1999), 146-156. MR 2002c:57014

[3] _ Quandle cohomology and state-sum invariants of knotted curves and surfaces, preprint.

[4] J. S. Carter, D. Jelsovsky, S. Kamada, and M. Saito, Computations of quandle cocycle invariants of knotted curves and surfaces, Adv. in Math. 157 (2001), 36-94. MR 2001m:57009]

[5] _ Quandle homology groups, their Betti numbers, and virtual knots, J. Pure Appl. Algebra 157 (2001), 135-155. MR 2002f:57010

[6] J. S. Carter, S. Kamada, and M. Saito, Geometric interpretations of quandle homology, J. Knot Theory Ramifications 10 (2001), 345-386. MR 2002h:57009

[7] Diagrammatic computations for quandles and cocycle knot invariants, preprint.

[8] J. S. Carter and M. Saito, Canceling branch points on projections of surfaces in 4-space, Proc. Amer. Math. Soc. 116 (1992), 229-237. MR 93i:57029

[9] _ Normal Euler classes of knotted surfaces and triple points on projections, Proc. Amer. Math. Soc. 125 (1997), 617-623. MR 97d:57030

[10] Knotted surfaces and their diagrams, Mathematical Surveys and Monographs, 55. Amer. Math. Soc., Providence, RI, 1998. MR 98m:57027

[11] T. Cochran, Ribbon knots in $S^{4}$, J. London Math. Soc. 28 (1983), 563-576. MR 85k:57019

[12] R. Fenn and C. Rourke, Racks and links in codimension two, J. Knot Theory Ramifications 1 (1992), 343-406. MR 94e:57006

[13] R. H. Fox, Metacyclic invariants of knots and links, Canad. J. Math. 22 (1970), 193-201. MR 41:6197; MR 48:9697

[14] D. L. Goldsmith and L. H. Kauffman, Twist spinning revisited, Trans. Amer. Math. Soc. 239 (1978), 229-251. MR 81f:57016

[15] A. Inoue, Quandle homomorphisms of knot quandles to Alexander quandles, J. Knot Theory Ramifications 10 (2001), 813-821. MR 2002e:57008

[16] D. Joyce, A classifying invariant of knots, the knot quandle, J. Pure Appl. Algebra 23 (1982), 37-65. MR 83m:57007 
[17] T. Kanenobu and A. Shima, Two filtrations of ribbon 2-knots, Topology Appl. 121 (2002), $143-168$.

[18] A. Kawauchi, T. Shibuya, and S. Suzuki, Descriptions on surfaces in four-space I, Normal forms, Math. Sem. Notes, Kobe Univ. 10 (1982), 75-125. MR 84d:57017

[19] A. Kawauchi, A Survey of Knot Theory, Birkhäuser Verlag, Basel, 1996. MR 97k:57011

[20] R. A. Litherland, Deforming twist-spun knots, Trans. Amer. Math. Soc. 250 (1979), 311-331. MR 80i:57015

[21] R. A. Litherland and S. Nelson, The Betti numbers of some finite racks, preprint.

[22] Y. Marumoto and Y. Nakanishi, A note on the Zeeman theorem, Kobe J. Math. 8 (1991), 67-71. MR 92h:57039

[23] S. Matveev, Distributive groupoids in knot theory, Mat. Sb. (N.S.) 119(161) (1982), 78-88, 160 (Russian); English transl., Math. USSR Sb. 47 (1984), 73-83. MR 84e:57008

[24] T. Mochizuki, Some calculations of cohomology groups of finite Alexander quandles, preprint.

[25] D. Roseman, Reidemeister-type moves for surfaces in four-dimensional space, in Banach Center Publications 42 (1998), Knot theory, 347-380. MR 99f:57029

[26] C. P. Rourke and B. J. Sanderson, Introduction to piecewise-linear topology, Ergebn. Math. U. ihrer Grenzgeb. Bd. 69, Springer-Verlag, Berlin-Heidelberg-New York, 1972. MR 50:3236

[27] $\longrightarrow$, There are two 2-twist-spun trefoils, preprint.

[28] S. Satoh, On non-orientable surfaces in 4-space which are projected with at most one triple point, Proc. Amer. Math. Soc. 128 (2000), 2789-2793. MR 2000m:57034

[29] _ Lifting a generic surface in 3-space to an embedded surface in 4-space, Topology Appl. 106 (2000), 103-113. MR 2001h:57028

[30] Minimal triple point numbers of some non-orientable surface-links, Pacific J. Math. 197 (2001), 213-221. MR 2001m:57031

[31] Surface diagrams of twist-spun 2-knots, J. Knot Theory Ramifications 11 (2002), 413-430. MR 2003e:57041

[32] A. Shima, Knotted 2-spheres whose projections into 3-space contain at most two triple points are ribbon 2-knots, preprint.

[33] _ Colorings and Alexander polynomials for ribbon 2-knots, J. Knot Theory Ramifications 11 (2002), 403-412.

[34] T. Yajima, On simply knotted spheres in $R^{4}$, Osaka J. Math. 1 (1964), 133-152. MR 30:2500

[35] E. C. Zeeman, Twisting spun knots, Trans. Amer. Math. Soc. 115 (1965), 471-495. MR 33:3290

Department of Mathematics, Chiba University, Inage, Chiba, 263-8522, Japan

E-mail address: satoh@math.s.chiba-u.ac.jp

Department of Mathematics, Tokai University, 1117 Kitakaname, Hiratuka, KanaGAWA, 259-1292, JAPAN

E-mail address: shima@keyaki.cc.u-tokai.ac.jp 